\title{
Etude biochimique d'un fromage de chèvre à croûte fleurie fabriqué à l'aide d'extraits coagulants microbiens
}

\author{
par \\ Anna POLYCHRONIADOU et K.S. MANOLKIDIS
}

\section{Ré s u mé}

Trois extraits coagulants microbiens, Noury Rennet (Mucor pusillus, Lindt), Rennilase (Mucor miehei) et Suparen (Endothia parasitica) ont été essayés pour la fabrication d'un fromage de chèvre à croûte fleurie. La présure animale a été utilisée comme témoin.

Les fromages ont été suivis pendant l'affinage et la conservation par l'intermédiaire d'analyses chimiques et organoleptiques. La vitesse et l'intensité de la protéolyse différaient significativement entre les quatre fromages. Des différences existaient aussi relativement à la concentration en acides gras et en acides aminés libres. Pourtant les qualités organoleptiques des fromages affinés étaient semblables. Ils avaient tous un goût agréable, piquant et une belle apparence. Aucun défaut n'a été décelé. Les trois extraits essayés possèdent une bonne aptitude à la fabrication de ce fromage de chèvre.

Mots clés : Fromage de chèvre - Extraits coagulants microbiens Affinage - Croûte fleurie - Protéolyse - Acides aminés - Acides gras.

\section{S u m m a r y}

Manufacture of a goat CHEese With SURFace FloRa USING SOME MICROBIAL COAGULANTS

Three microbial rennets, Noury Rennet (Mucor pusillus, Lindt), Rennilase (Mucor miehei) and Suparen (Endothia parasitica) were assessed, in comparison with calf rennet, for the manufacture of a

Laboratoire de Technologie Laitière, Université de Thessalonique - 54636 Thessalonique (Grèce). 
goat cheese ripened under the influence of a surface flora (Penicillium caseicolum).

Cheeses were followed throughout ripening by chemical and organoleptic analyses. Rate, and extent of proteolysis differed significantly among cheeses. There were significant differences for free amino acid and fatty acid content too. However, the mean scores for flavour and body texture were similar for all mature cheeses. No bitterness, off-flavour or body defect was noted by any member of the taste panel. There were no apparent differences in yield between experimental and control cheeses. Noury Rennet, Rennilase and Suparen are well suited for the manufacture of this type of goat cheese.

Key words: Goat cheese - Microbial coagulants - Ripening Surface flora - Proteolysis - Amino acids - Fatty acids.

\section{INTRODUCTION}

La quantité de lait de chèvre produit en Grèce atteignait en 1980 414000 tonnes par an (F.I.L., 1983). Il est utilisé, en mélange avec du lait de brebis, à la préparation des fromages Feta et Télémé. Etant donné que le relief du sol et le climat en Grèce sont favorables pour l'élévage de chèvres, la quantité de lait produit pourrait augmenter au profit de la population rurale. Cet accroissement de quantité pourrait être utilisé pour la préparation de produits nouveaux pour le marché grec, comme le sont les fromages " pur chèvre » à croûte fleurie.

En même temps, la production universelle de la présure devient de plus en plus incapable à couvrir la demande et son prix augmente continuellement.

On a donc essayé de produire un fromage de chèvre à l'aide d'extraits coagulants microbiens. Le présent article rapporte les résultats de cette étude.

\subsection{Lait}

\section{MATERIEL ET METHODES}

Le lait de chèvre utilisé provenait du ramassage d'une fromagerie de la région de Thessalonique. Il était thermisé à $63^{\circ} \mathrm{C}$ pendant $10 \mathrm{~min}$.

\subsection{Ferments lactiques}

Le lait était additionné de ferments lactiques mésophiles et thermophiles, cultivés séparément et ajoutés en quantités respectives de 2 à $1 \%(v / v)$. 
Les micro-organismes utilisés étaient : Streptococcus lactis et Str. cremoris (cultures lyophilisées, $\mathrm{n}^{\circ} 95$ et 170, Type 0, Chr. Hansen Laboratorium A/S, Dänemark) et Streptococcus thermophilus (culture lyophilisée, Wiesby Lab., W. Germany).

\subsection{Chlorure de calcium}

Chaque litre de lait était additionné de $0,3 \mathrm{~g} \mathrm{CaCl}_{2}$ sous forme d'une solution $40 \%(\mathrm{w} / \mathrm{v})$.

\subsection{Enzymes coagulants}

Les extraits coagulants utilisés étaient :

(a) Présure Hansen (Chr. Hansen Laboratorium A/S, Dänemark). Poudre soluble à titre 1:100000. Elle était utilisée comme préparation témoin.

(b) "Noury Rennet " (AKZO Chemie Nederland bv) provenant de Mucor pusillus, Lindt. Poudre soluble à titre 1:220000.

(c) "Rennilase » (Novo Industri A/S, Dänemark) provenant de Mucor miehei. Poudre soluble à titre 1: 10700.

(d) "Suparen » (Pfizer Ltd, U.K.) provenant de Endothia parasitica. Poudre soluble à titre $1: 60000$.

A l'issue des essais préliminaires, les quantités suivantes de chacun d'eux étaient utilisées :

$\begin{array}{lllr}\text { Présure } & 50 \mathrm{mg} & \text { Rennilase } & 50 \mathrm{mg} \\ \text { Noury Rennet } & 10 \mathrm{mg} & \text { Suparen } & 125 \mathrm{mg}\end{array}$

afin que la durée du caillage de $5 \mathrm{~kg}$ de lait de chèvre soit identique pour les quatre enzymes dans nos conditions expérimentales.

\subsection{Moisissure superficielle}

La flore superficielle des fromages était produite par le développement des spores de Penicillium caseicolum (Wiesby Lab., W. Germany).

\subsection{Mode de fabrication des fromages}

Vingt kilogrammes de lait caprin entier étaient thermisés, refroidis à $28^{\circ} \mathrm{C}$, additionnés de chlorure de calcium et ensemencés de ferments lactiques. Lorsque l'acidité atteignait $21^{\circ} \mathrm{D}(\mathrm{pH}$ environ 6,4 ) le lait était partagé en quatre bassines à chacune desquelles était ajoutée la quantité appropriée d'un des quatre extraits coagulants.

Le caillage avait lieu à $28^{\circ} \mathrm{C}$ et se poursuivait pendant $90 \mathrm{~min}$. Le caillé était découpé et déposé dans des moules cylindriques (diamètre : $10 \mathrm{~cm}$, hauteur : $10 \mathrm{~cm}$ ) lorsque l'acidité du sérum atteignait 
$16^{\circ} \mathrm{D}$. L'égouttage était spontané, aidé par retournements des fromages à $28^{\circ} \mathrm{C}$ pour les deux premières heures et, ensuite, à $22-24^{\circ} \mathrm{C}$.

Après $24 \mathrm{~h}$ le caillé, démoulé, était ensemencé par vaporisation d'une suspension de spores de Penicillium caseicolum et saupoudré de sel. L'affinage était accompli dans des hâloirs d'une température de $12^{\circ} \mathrm{C}$ et d'une humidité relative de l'ordre de 80 à $90 \%$. Il durait 30 jours.

Les fromages affinés étaient enveloppés dans des feuilles d'aluminium et conservés à $4^{\circ} \mathrm{C}$ pendant 30 à 45 jours.

La fabrication était répétée huit fois. Les fromages étaient caractérisés par deux lettres, selon l'extrait coagulant utilisé pour leur fabrication. Ainsi les fromages PR, NR, RE et SU ont été fabriqués respectivement à l'aide de présure, Noury Rennet, Rennilase et Suparen.

\subsection{Analyse des fromages}

Des analyses des fromages étaient effectuées à plusieurs reprises au cours de l'affinage. Chaque échantillon était le produit de broyage de la moitié d'un fromage, croûte comprise.

L'analyse comprenait la détermination de l'humidité et du sel selon les méthodes de la F.I.L. (normes $4: 1958$ et 17A : 1972), du $\mathrm{pH}$, de la matière grasse selon Gerber-van Gulik et de l'azote total selon Kjeldahl. On déterminait aussi l'azote soluble à $\mathrm{pH}$ 4,6 (NCN), l'azote soluble à $12 \%$ d'acide trichloroacétique (NPN), l'azote soluble dans l'eau à $45^{\circ} \mathrm{C}(\mathrm{SN})$ et l'ammoniac selon Mrowetz (1979).

La détermination des acides aminés libres dans les échantillons de fromages affinés était accomplie selon Spackman et coll. (1958) à l'aide d'un anlyseur automatique Unichrom Beckman. Les solutions des acides aminés étaient préparées selon Kosikowski (1951).

Les acides gras libres des fromages affinés étaient extraits (Nieuwenhof et Hup, 1971), transformés en esters méthyliques (Iyer et coll., 1967) et, finalement, séparés et dosés par chromatographie en phase gazeuse à l'aide d'un chromatographe Hewlett Packard modèle 5750 .

L'électrophorèse des échantillons de fromages était effectuée sur un gel de polyacrylamide $5 \%$ en tampon veronal $(0,05 \mathrm{M} ; \mathrm{pH} 8,6)$ contenant 5,0 M d'urée, selon les conditions décrites par Assenat (1967).

\subsection{Examen organoleptique des fromages}

Dès le $20^{\text {e }}$ jour de l'affinage un examen organoleptique des fromages avait lieu en même temps que leur analyse chimique. La saveur et l'arôme, la texture et la fermeté de la pâte, la couleur et l'apparence générale, ainsi que l'intensité du goût salé étaient évalués 
par un groupe de 10 juges se servant d'une fiche de cotation. Cette dernière était composée selon les règles et les suggestions de Nelson et Trout (1965). Les facteurs, classés par ordre de leur contribution au score final, ainsi que l'étendue des échelles de graduation sont donnés au tableau 1.

$$
\text { TABLEAU } 1 \text { - TABLE } 1
$$

Fiche de cotation pour l'appréciation organoleptique

des fromages à examiner

Score card for the organoleptic evaluation of the cheeses under examination

\begin{tabular}{l|c}
\hline \multicolumn{1}{c|}{ Facteur organoleptique } & $\begin{array}{c}\text { Etendue de l'échelle } \\
\text { de graduation (points) }\end{array}$ \\
\hline Saveur et arôme & $35-45$ \\
Texture et fermeté de la pâte & $30-35$ \\
Couleur et apparence générale & $10-15$ \\
Sel & $3-5$ \\
\hline Total & $78-100$ \\
\hline
\end{tabular}

\subsection{Analyse statistique des résultats}

Les résultats des analyses chimiques, ainsi que ceux des examens organoleptiques, ont fait l'objet d'analyses de variance. Les moyennes étaient comparées à l'aide du test F. Pour les moyennes différant au seuil 0,05 on calculait la plus petite différence significative.

\section{RESULTATS ET DISCUSSION}

\subsection{Description des fromages}

Les fromages affinés avaient la forme d'un cylindre plat de $10 \mathrm{~cm}$ de diamètre et $2,5 \mathrm{~cm}$ d'épaisseur. Leur surface était couverte des filaments mycéliens de Pénicillium, formant une couche d'environ $1 \mathrm{~mm}$ d'épaisseur. La pâte des fromages était molle, onctueuse, blanchâtre. Aucune différence n'a été remarquée entre les fromages préparés à l'aide des quatre extraits différents. 


\subsection{Rendement fromager}

Les rendements fromagers étaient pratiquement identiques. Le poids moyen des fromages au $3^{\mathrm{e}}$ jour, calculé pour $100 \mathrm{~kg}$ de lait, était $15,06(7,88) \mathrm{kg}$ pour $\mathrm{PR}, 15,11(7,95) \mathrm{kg}$ pour $\mathrm{NR}, 15,37(8,10) \mathrm{kg}$ pour RE et $15,06(7,96) \mathrm{kg}$ pour SU. Les chiffres entre parenthèses donnent le poids sec.

Le rendement fromager semble pareil ou supérieur à ceux rapportés par Ramet et coll. (1969) et Ramet et Alais (1972) pour des fromages de type Camembert fabriqués avec des coagulants fongiques à partir de lait de vache. Mais il est significativement supérieur si l'on considère le poids sec. Cela peut être attribué à la composition du lait de chèvre, plus riche en extrait sec et en matière grasse que celui de vache.

Ces résultats doivent être confirmés par des fabrications en échelle industrielle.

\subsection{Composition des fromages}

Le tableau 2 présente la composition des fromages expérimentaux au cours de l'affinage.

Les résultats des analyses chimiques donnés sont la moyenne des huit prélèvements correspondant aux huit fabrications de chaque fromage.

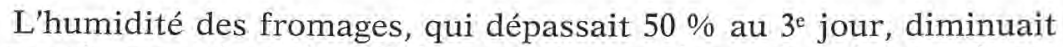
progressivement jusqu'au $30^{\mathrm{e}}$ jour et elle restait par la suite au niveau de 40 à $42 \%$. Aucune différence notable n'a été constatée entre les fromages préparés au moyen des différents extraits coagulants.

La proportion en matière grasse ne changeait pas au cours de l'affinage. Elle était identique pour les quatre fromages expérimentaux et toujours élevée (de 60,01 à 60,95\%).

La teneur en chlorure de sodium, calculée en pourcentage de la phase aqueuse, ne présentait pas de différences entre les fromages. Elle augmentait progressivement à cause de la diminution simultanée de l'humidité.

Quant au $\mathrm{pH}$ des fromages, il montrait un accroissement très prononcé pendant les 30 premiers jours de l'affinage (tab. 2). Ce fait peut être attribué tant à la production d'ammoniac, produit final de la dégradation des protéines, qu'à la consommation de l'acide lactique par la flore superficielle, abondamment développée pendant cette période. L'augmentation du $\mathrm{pH}$ après les 30 jours était moins intense, mais toujours significative. Ainsi, au $60^{\mathrm{e}}$ jour, il atteignait la neutralité, qu'il dépassait au $75^{\mathrm{e}}$ jour. A ce stade, commençait la décomposition des fromages. L'évolution du $\mathrm{pH}$ des quatre fromages expérimentaux avait lieu de la même manière et les diffé- 
TABLEAU 2 - TABLE 2

Composition des fromages fabriqués à l'aide de présure animale (PR),

de Noury Rennet (NR), de Rennilase (RE) et de Suparen (SU)

Composition of cheeses produced using calf rennet $(P R)$,

Noury Rennet (NR), Rennilase (RE) and Suparen (SU) as milk coagulants

\begin{tabular}{|c|c|c|c|c|c|c|c|c|}
\hline & \multirow{2}{*}{ Fromage } & \multicolumn{7}{|c|}{ Durée de l'affinage (jours) } \\
\hline & & 3 & 10 & 20 & 30 & 45 & 60 & 75 \\
\hline Humidité \% & $\begin{array}{l}\text { PR } \\
\text { NR } \\
\text { RE } \\
\text { SU }\end{array}$ & $\begin{array}{l}51,36 \\
51,12 \\
51,91 \\
50,71\end{array}$ & $\begin{array}{l}48,01 \\
47,76 \\
48,27 \\
48,75\end{array}$ & $\begin{array}{l}45,38 \mathrm{a} \\
45,58 \mathrm{a} \\
44,90 \mathrm{ab} \\
43,85^{\mathrm{b}}\end{array}$ & $\begin{array}{l}43,75 \mathrm{a} \\
43,18 \mathrm{ab} \\
42,13 \mathrm{~b} \\
43,11 \mathrm{ab}\end{array}$ & $\begin{array}{l}42,44 \\
43,15 \\
41,69 \\
42,19\end{array}$ & $\begin{array}{l}42,00 \mathrm{ab} \\
41,15 \mathrm{ab} \\
40,83 \mathrm{a} \\
42,32 \mathrm{~b}\end{array}$ & $\begin{array}{l}42,19 \mathrm{a} \\
40,50 \mathrm{~b} \\
41,01 \mathrm{ab} \\
41,86 \mathrm{a}\end{array}$ \\
\hline $\begin{array}{l}\text { Matière grasse } \\
\text { (g p. } 100 \mathrm{~g} \text { de matière } \\
\text { sèche) }\end{array}$ & $\begin{array}{l}\text { PR } \\
\text { NR } \\
\text { RE } \\
\text { SU }\end{array}$ & $\begin{array}{l}60,73 \\
60,50 \\
60,43 \\
60,01\end{array}$ & $\begin{array}{l}60,53 \\
60,72 \\
60,44 \\
60,46\end{array}$ & $\begin{array}{l}60,36 \\
60,39 \\
60,62 \\
60,35\end{array}$ & $\begin{array}{l}60,03 \\
60,13 \\
60,06 \\
60,07\end{array}$ & $\begin{array}{l}60,95 \\
60,27 \\
60,92 \\
60,45\end{array}$ & $\begin{array}{l}60,02 \\
60,82 \\
60,83 \\
60,27\end{array}$ & $\begin{array}{l}60,27 \\
60,61 \\
60,93 \\
60,95\end{array}$ \\
\hline $\begin{array}{l}\text { Azote total } \\
\text { (g p. } 100 \mathrm{~g} \text { de matière } \\
\text { sèche) }\end{array}$ & $\begin{array}{l}\text { PR } \\
\text { NR } \\
\text { RE } \\
\text { SU }\end{array}$ & $\begin{array}{l}5,93 \\
6,07 \\
6,02 \\
5,99\end{array}$ & $\begin{array}{l}6,02 \\
6,05 \\
5,99 \\
5,94\end{array}$ & $\begin{array}{l}5,82 \\
5,94 \\
5,89 \\
5,79\end{array}$ & $\begin{array}{l}5,75 \\
5,84 \\
5,91 \\
5,89\end{array}$ & $\begin{array}{l}5,70 \\
5,81 \\
5,77 \\
5,83\end{array}$ & $\begin{array}{l}5,72 \\
5,79 \\
5,74 \\
5,80\end{array}$ & $\begin{array}{l}5,71 \\
5,83 \\
5,68 \\
5,85\end{array}$ \\
\hline $\begin{array}{l}\mathrm{NaCl} \\
\text { (g p. } 100 \text { g de la phase } \\
\text { aqueuse) }\end{array}$ & $\begin{array}{l}\text { PR } \\
\text { NR } \\
\text { RE } \\
\text { SU }\end{array}$ & $\begin{array}{l}2,48 \\
2,21 \\
2,28 \\
2,64\end{array}$ & $\begin{array}{l}2,76 \\
2,46 \\
2,70 \\
2,78\end{array}$ & $\begin{array}{l}3,47 \\
3,47 \\
3,56 \\
3,25\end{array}$ & $\begin{array}{l}4,05 \\
3,83 \\
3,75 \\
3,65\end{array}$ & $\begin{array}{l}4,42 \\
3,93 \\
3,95 \\
3,98\end{array}$ & $\begin{array}{l}4,55 \mathrm{a} \\
3,96 \mathrm{~b} \\
4,39 \mathrm{ab} \\
4,01 \mathrm{ab}\end{array}$ & $\begin{array}{l}4,69 \mathrm{a} \\
4,00 \mathrm{~b} \\
4,56 \mathrm{ab} \\
4,05 \mathrm{~b}\end{array}$ \\
\hline $\mathrm{pH}$ & $\begin{array}{l}\text { PR } \\
\text { NR } \\
\text { RE } \\
\text { SU }\end{array}$ & $\begin{array}{l}4,54 \mathrm{ab} \\
4,63 \mathrm{~b} \\
4,45 \mathrm{a} \\
4,45 \mathrm{a}\end{array}$ & $\begin{array}{l}5,33 \\
5,26 \\
5,27 \\
5,34\end{array}$ & $\begin{array}{l}5,76 \mathrm{a} \\
5,68 \mathrm{a} \\
5,73 \mathrm{a} \\
5,52 \mathrm{~b}\end{array}$ & $\begin{array}{l}6,64 \mathrm{a} \\
6,31 \mathrm{~b} \\
6,30 \mathrm{~b} \\
6,30 \mathrm{~b}\end{array}$ & $\begin{array}{l}6,58 \\
6,53 \\
6,57 \\
6,52\end{array}$ & $\begin{array}{l}6,83 \mathrm{a} \\
6,86 \mathrm{ab} \\
6,95 \mathrm{~b} \\
7,09 \mathrm{c}\end{array}$ & $\begin{array}{l}7,20 \\
7,15 \\
7,30 \\
7,37\end{array}$ \\
\hline
\end{tabular}

a, b, c : les valeurs d'une même colonne, portant une lettre différente, diffèrent significativement au seuil de $\mathrm{P}=0,05$. 
rences constatées entre eux dépassaient quelquefois à peine la plus petite différence significative $(\mathrm{P}<0,05)$.

Le pourcentage en azote total restait pratiquement constant au cours de la maturation et ne montrait aucune différence significative entre les fromages fabriqués à l'aide d'extraits différents. Par contre, l'évolution des fractions azotées solubles était très marquée et variait entre les fromages expérimentaux (tab. 3).

La protéolyse commençait dès les premiers jours. Après 10 jours, 13 à $16 \%$ de l'azote total n'était pas précipitable à pH 4,6. Vers le $30^{\mathrm{e}}$ jour, quand les fromages avaient atteint leur maturité, ce pourcentage qui montait à $20 \%$, était pour une large part de nature non-protéique. Pendant cette période, l'action des protéases coagulantes était complétée par celle des protéinases et des peptidases des streptocoques lactiques (Exterkate, 1975; Desmazeaud et Juge, 1976 ; Thomas et Mills, 1981) conduisant à la production d'acides aminés et de peptides à bas poids moléculaire.

La dégradation des substances azotées continuait tout le long de l'affinage. Le rôle propre de la flore superficielle ne peut pas être ignoré : Gripon et coll. (1977) ont montré que l'activité protéolytique de l'endopeptidase neutre de Penicillium caseicolum est si grande qu'elle dissimule celle des protéases coagulantes et des ferments lactiques. Noomen (1983) a récemment constaté que l'action de $P$. caseicolum est limitée à la surface des fromages. Ce qui est certain c'est que ces différentes enzymes protéolytiques agissent de façon synergique et l'ammoniac, produit final de la dégradation des substances azotées, est libéré en quantités importantes (tab. 3) et diffuse vers le centre du fromage. Il s'ensuit une modification graduelle du $\mathrm{pH}$ qui favorise le changement de consistance du fromage (Noomen, 1983).

Bien que l'évolution de la protéolyse était semblable pour les quatre fromages expérimentaux, on a observé des différences au niveau de la vitesse et de l'intensité avec lesquelles elle se réalisait. Les proportions des diverses formes d'azote soluble devenaient significativement différentes $(\mathrm{P}<0,05)$ spécialement après le $30^{\mathrm{e}}$ jour. Le fromage PR montrait la protéolyse la plus réduite, suivi par NR, RE et SU. Ceci est en concordance avec les résultats de Maubois et Mocquot (1969), Ramet et coll. (1969) et Ramet et Alais (1972) qui étudiant l'aptitude de protéases fongiques dans la fabrication de fromages à pâte molle, ont constaté que la protéolyse se développe plus rapidement avec ces protéases qu'avec la présure. L'accélération de la solubilisation de la caséine par les coagulants microbiens a aussi été relevée pour d'autres types de fromages (Nelson, 1969; Vanderpoorten et Weckx, 1972; Green et Stackpoole, 1975).

Les peptides à poids moléculaire élevé (SN-NPN) constituent la majeure partie de l'azote hydrosoluble et sont accumulés plus rapidement aux fromages NR, RE et SU. Ce fait peut être attribué au 


\section{TABLEAU $3-T A B L E 3$}

Variations de la quantité des diverses formes d'azote soluble des fromages au cours de l'affinage (g p. $100 \mathrm{~g}$ d'azote total)

Vatiations in the quantity of the different soluble nitrogen forms of cheeses during ripening (as percentage of total nitrogen)

\begin{tabular}{|c|c|c|c|c|c|c|c|c|}
\hline & \multirow{2}{*}{ Fromage } & \multicolumn{7}{|c|}{ Durée de l'affinage (jours) } \\
\hline & & 3 & 10 & 20 & 30 & 45 & 60 & 75 \\
\hline $\begin{array}{l}\text { Azote non protéique } \\
(\mathrm{NPN})\end{array}$ & $\begin{array}{l}\text { PR(1) } \\
\text { NR(1) } \\
\text { RE(1) } \\
\text { SU(1) }\end{array}$ & $\begin{array}{l}5,21 \\
5,67 \\
6,05 \\
6,41\end{array}$ & $\begin{array}{r}7,97 \mathrm{a} \\
9,75 \mathrm{~b} \\
11,31 \mathrm{c} \\
9,43 \mathrm{ab}\end{array}$ & $\begin{array}{l}11,12 \\
11,79 \\
12,52 \\
11,65\end{array}$ & $\begin{array}{l}17,03 \mathrm{a} \\
17,86 \mathrm{ab} \\
18,95 \mathrm{~b} \\
16,94 \mathrm{a}\end{array}$ & $\begin{array}{l}18,04 \mathrm{a} \\
23,79 \mathrm{~b} \\
22,04 \mathrm{c} \\
20,56 \mathrm{c}\end{array}$ & $\begin{array}{l}18,93 \mathrm{a} \\
24,17 \mathrm{~b} \\
22,33 \mathrm{c} \\
20,96 \mathrm{c}\end{array}$ & $\begin{array}{l}25,00 \\
25,03 \\
25,73 \\
25,65\end{array}$ \\
\hline $\begin{array}{l}\text { Azote non caséique } \\
(\mathrm{NCN})\end{array}$ & $\begin{array}{l}\text { PR } \\
\text { NR } \\
\text { RE } \\
\text { SU }\end{array}$ & $\begin{array}{l}7,36 \\
9,71 \\
8,74 \\
9,24\end{array}$ & $\begin{array}{l}13,00 \\
16,08 \\
16,60 \\
16,22\end{array}$ & $\begin{array}{l}18,02 \\
19,77 \\
20,70 \\
19,13\end{array}$ & $\begin{array}{l}18,89 a \\
23,59 \mathrm{~b} \\
21,73 a b \\
25,59 \mathrm{~b}\end{array}$ & $\begin{array}{l}23,84 \mathrm{a} \\
32,86 \mathrm{~b} \\
32,52 \mathrm{~b} \\
31,93 \mathrm{~b}\end{array}$ & $\begin{array}{l}27,31 \mathrm{a} \\
34,65^{\mathrm{b}} \\
34,61^{\mathrm{b}} \\
32,63^{\mathrm{b}}\end{array}$ & $\begin{array}{l}33,79 \mathrm{a} \\
38,46 \mathrm{~b} \\
38,52 \mathrm{~b} \\
36,38 \mathrm{ab}\end{array}$ \\
\hline Azote soluble ( $\mathrm{SN}$ ) & $\begin{array}{l}\text { PR } \\
\text { NR } \\
\text { RE } \\
\text { SU }\end{array}$ & $\begin{array}{l}6,16 \\
6,21 \\
6,83 \\
7,03\end{array}$ & $\begin{array}{l}12,02 \\
13,85 \\
13,97 \\
12,90\end{array}$ & $\begin{array}{l}20,45 \mathrm{a} \\
25,51 \mathrm{~b} \\
22,99 \mathrm{ab} \\
23,07 \mathrm{ab}\end{array}$ & $\begin{array}{l}40,22 \mathrm{a} \\
45,59 \mathrm{~b} \\
45,80 \mathrm{~b} \\
45,32 \mathrm{~b}\end{array}$ & $\begin{array}{l}41,40 \mathrm{a} \\
55,08^{\mathrm{b}} \\
52,46^{\mathrm{b}} \\
53,87 \mathrm{~b}\end{array}$ & $\begin{array}{l}57,38 \mathrm{a} \\
69,09 \mathrm{~b} \\
67,31 \mathrm{~b} \\
67,18^{\mathrm{b}}\end{array}$ & $\begin{array}{l}70,26 \mathrm{a} \\
83,65^{\mathrm{b}} \\
80,74 \mathrm{~b} \\
76,76^{\mathrm{c}}\end{array}$ \\
\hline Ammoniac & $\begin{array}{l}\text { PR } \\
\text { NR } \\
\text { RE } \\
\text { SU }\end{array}$ & $\begin{array}{l}0,89 \\
0,84 \\
0,86 \\
0,85\end{array}$ & $\begin{array}{l}2,22 \\
2,21 \\
2,39 \\
2,32\end{array}$ & $\begin{array}{l}3,90 \mathrm{a} \\
4,01 \mathrm{~b} \\
4,36 \mathrm{c} \\
3,92 \mathrm{a}\end{array}$ & $\begin{array}{l}6,30 \mathrm{a} \\
6,76 \mathrm{~b} \\
6,60 \mathrm{bc} \\
6,50 \mathrm{c}\end{array}$ & $\begin{array}{l}8,19 a \\
9,04 b \\
8,96 b \\
8,99 b\end{array}$ & $\begin{array}{l}10,47 \mathrm{a} \\
12,23 \mathrm{~b} \\
11,32 \mathrm{c} \\
11,10 \mathrm{c}\end{array}$ & $\begin{array}{l}12,80 \mathrm{a} \\
15,27 \mathrm{~b} \\
15,62 \mathrm{c} \\
14,53 \mathrm{~d}\end{array}$ \\
\hline
\end{tabular}

(1) Fromages fabriqués à l'aide de présure animale (PR), de Noury Rennet (NR), de Rennilase (RE) et de Suparen (SU). a, b, c, d : les valeurs d'une même colonne, portant une lettre différente, diffèrent significativement au seuil de $P=0,05$. 
pH élevé des fromages expérimentaux, qui favorise l'action des coagulants d'origine fongique (Alais et Novak, 1968; Alais et Lagrange, 1972).

Les électrophorégrammes des quatre fromages au $3^{\mathrm{e}}$ et $30^{\mathrm{e}}$ jour de l'affinage (fig. 1) confirment les résultats des analyses chimiques. Chaque protéinase a hydrolysé les caséines d'une manière différente et les produits de dégradation ne sont pas les mêmes. Le Noury Rennet est celui qui ressemble le plus à la présure, son action pro-

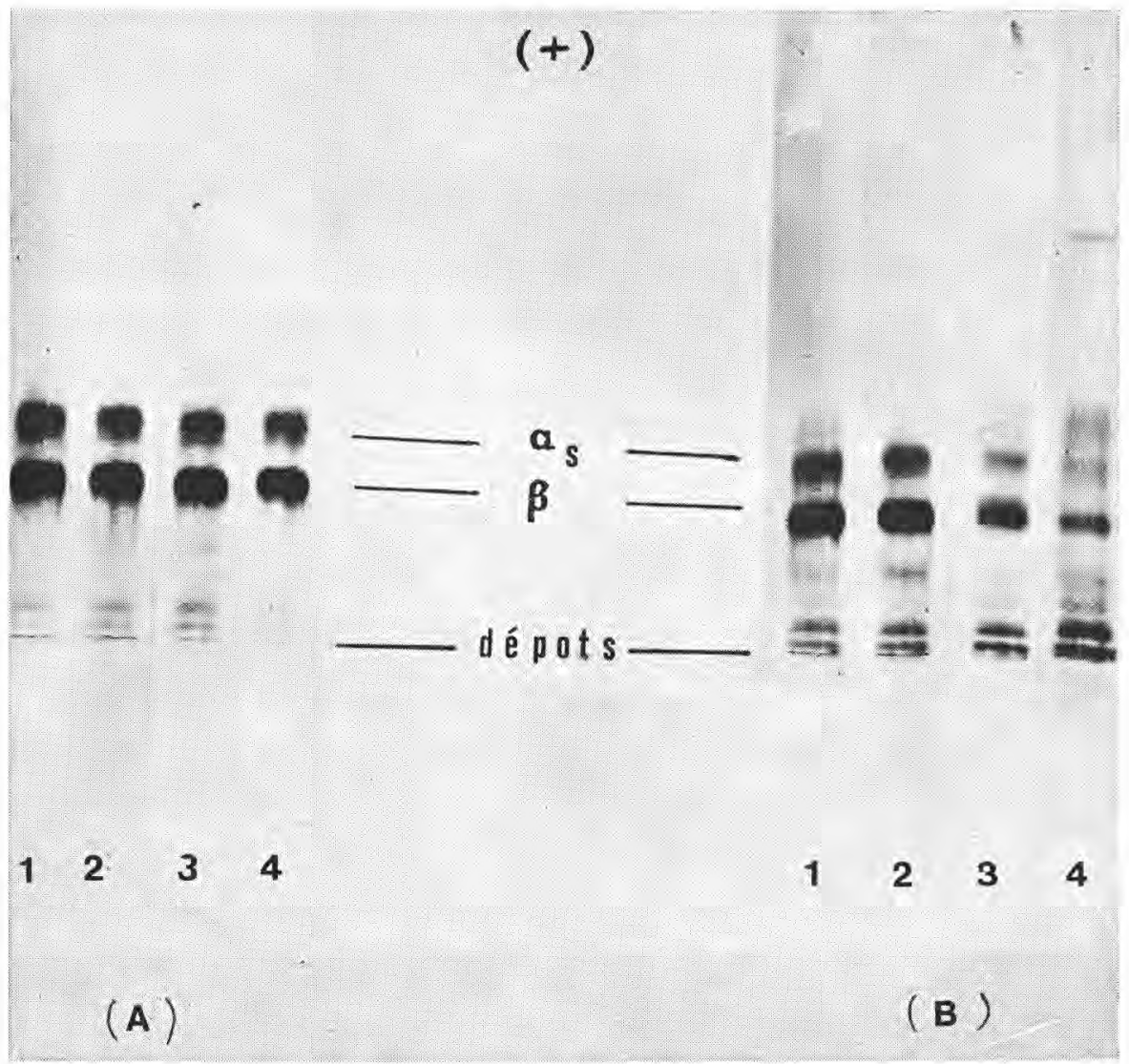

fig. 1

Electrophorèse sur gel de polyacrylamide de fromages préparés avec (1) présure animale, (2) Noury Rennet, (3) Rennilase et (4) Suparen après (A) et (B) 30 jours d'affinage.

Polyacrylamide gel electrophoresis of cheeses made with (1) calf rennet, (2) Noury Rennet, (3) Rennilase and (4) Suparen, after $a$ (A) 3 and (B) 30 days ripening. 
téolytique étant plus régulière et plus modérée. La Rennilase et le Suparen donnent un diagramme différent.

La composition en acides aminés libres des fromages au 30 jour d'affinage est présentée au tableau 4. Le fromage RE ressemble le plus au PR, mais la concentration de la proline était inférieure et celles de la sérine et de l'acide glutamique supérieures. Les résultats étaient différents pour les fromages NR et SU. La concentration en leucine, méthionine, proline et acide glutamique, ainsi que la quantité totale des acides aminés dépassaient significativement celles du fromage PR.

La lipolyse des fromages affinés était estimée par la détermination des acides gras libres (tab. 5). Les différences entre les quatre

\section{TABLEAU 4-TABLE 4}

Acides aminés libres (mg/100 g de matière sèche) des fromages affinés, 30 jours après leur fabrication

Free amino acid content ( $\mathrm{mg} / 100 \mathrm{~g}$ dry matter) of the 30-days-old cheeses

\begin{tabular}{|c|c|c|c|c|}
\hline \multirow{2}{*}{ Acides aminés } & \multicolumn{4}{|c|}{ Fromages } \\
\hline & $\mathrm{PR}(1)$ & NR(1) & $\mathrm{RE}(1)$ & $\mathrm{SU}(1)$ \\
\hline $\begin{array}{l}\text { Acide aspartique } \\
\text { Théonine } \\
\text { Sérine } \\
\text { Acide glutamique } \\
\text { Proline } \\
\text { Glycine } \\
\text { Alanine } \\
\text { Valine } \\
\text { Méthionine } \\
\text { Isoleucine } \\
\text { Leucine } \\
\text { Tyrosine } \\
\text { Phénylalanine } \\
\text { Citrulline } \\
\text { Lysine } \\
\text { Histidine } \\
\text { Arginine }\end{array}$ & $\begin{array}{l}32,8 \\
15,5 \mathrm{a} \\
32,3 \mathrm{a} \\
75,7 \mathrm{a} \\
39,1 \mathrm{a} \\
7,6 \\
25,7 \\
26,1 \mathrm{ab} \\
20,1 \mathrm{a} \\
31,5 \mathrm{a} \\
66,2 \mathrm{a} \\
29,6 \\
42,9 \\
13,7 \mathrm{a} \\
43,8 \\
21,1 \\
15,9\end{array}$ & $\begin{array}{c}29,1 \\
14,7 \mathrm{a} \\
31,8 \mathrm{a} \\
147,7 \mathrm{~b} \\
45,9 \mathrm{~b} \\
6,9 \\
28,6 \\
28,5 \mathrm{~b} \\
26,1 \mathrm{~b} \\
31,9 \mathrm{a} \\
92,5 \mathrm{bc} \\
33,3 \\
53,2 \\
23,3 \mathrm{~b} \\
42,6 \\
25,9 \\
16,8\end{array}$ & $\begin{array}{c}31,6 \\
12,9 \mathrm{a} \\
50,0 \mathrm{~b} \\
113,1 \mathrm{c} \\
25,5 \mathrm{c} \\
4,3 \\
20,4 \\
22,5 \mathrm{a} \\
21,8 \mathrm{a} \\
28,4 \mathrm{a} \\
71,6 \mathrm{ab} \\
30,5 \\
44,0 \\
12,9 \mathrm{a} \\
47,7 \\
16,5 \\
13,1\end{array}$ & $\begin{array}{c}25,4 \\
38,9 \mathrm{~b} \\
37,7 \mathrm{a} \\
122,5 \mathrm{~d} \\
52,1 \mathrm{~d} \\
7,6 \\
20,8 \\
33,0 \mathrm{c} \\
35,6 \mathrm{c} \\
43,5 \mathrm{~b} \\
95,1 \mathrm{c} \\
38,6 \\
59,7 \\
13,7 \mathrm{a} \\
46,3 \\
23,1 \\
15,3\end{array}$ \\
\hline Total & 539,6 & 678,8 & 566,8 & 708,9 \\
\hline
\end{tabular}

(1) Fromages fabriqués à l'aide de présure animale (PR), de Noury Rennet (NR), de Rennilase (RE) et de Suparen (SU).

a, b, c, d : les valeurs d'une même ligne, portant une lettre différente, diffèrent significativement au seuil de $\mathrm{P}=0,05$. 


\section{TABLEAU $5-T A B L E 5$}

Acides gras libres (mg/100 $\mathrm{g}$ de matière sèche) des fromages affinés, 30 jours après leur fabrication

Free fatty acid content ( $\mathrm{mg} / 100 \mathrm{~g}$ dry matter) of the 30-days-old cheeses

\begin{tabular}{|c|c|c|c|c|}
\hline \multirow[t]{2}{*}{ Acides gras } & \multicolumn{4}{|c|}{ Fromages } \\
\hline & $\mathrm{PR}(1)$ & $\mathrm{NR}(1)$ & $\mathrm{RE}(1)$ & SU(1) \\
\hline $\left.\begin{array}{l}\mathrm{C}_{6: 0} \\
\mathrm{C}_{8: 0} \\
\mathrm{C}_{10: 0} \\
\mathrm{C}_{12: 0} \\
\mathrm{C}_{14: 0} \\
\mathrm{C}_{16: 0} \\
\mathrm{C}_{18: 0} \\
\mathrm{C}_{18: 1} \\
\mathrm{C}_{18: 2} \\
\mathrm{C}_{18: 3}\end{array}\right\}$ & $\begin{array}{r}337,6 \mathrm{a} \\
91,0 \mathrm{a} \\
189,6 \mathrm{a} \\
187,1 \mathrm{a} \\
713,6 \mathrm{a} \\
1164,6 \mathrm{a} \\
671,9 \mathrm{a} \\
865,2 \mathrm{a} \\
198,6 \mathrm{a}\end{array}$ & $\begin{array}{r}291,4 \mathrm{~b} \\
166,2 \mathrm{~b} \\
338,6 \mathrm{~b} \\
283,3 \mathrm{~b} \\
691,7 \mathrm{~b} \\
1471,5 \mathrm{~b} \\
954,8 \mathrm{~b} \\
1095,8 \mathrm{~b} \\
279,9 \mathrm{~b}\end{array}$ & $\begin{array}{r}600,5 \mathrm{c} \\
181,3 \mathrm{c} \\
232,4 \mathrm{c} \\
192,8 \mathrm{a} \\
794,7 \mathrm{c} \\
1671,9 \mathrm{c} \\
794,6 \mathrm{c} \\
871,3 \mathrm{a} \\
194,9 \mathrm{a}\end{array}$ & $\begin{array}{r}163,1 \mathrm{~d} \\
70,3 \mathrm{~d} \\
254,9 \mathrm{c} \\
203,8 \mathrm{c} \\
428,0 \mathrm{c} \\
932,0 \mathrm{~d} \\
472,5 \mathrm{~d} \\
361,6 \mathrm{c} \\
101,0 \mathrm{c}\end{array}$ \\
\hline Total & 4419,2 & 5573,2 & 5534,4 & 2987,2 \\
\hline
\end{tabular}

(1) Fromages fabriqués à l'aide de présure animale (PR), de Noury Rennet (NR), de Rennilase (RE) et de Suparen (SU).

a, b, c, d : les valeurs d'une même ligne, portant une lettre différente, diffèrent significativement au seuil de $\mathrm{P}=0,05$.

fromages expérimentaux dépassaient considérablement la plus petite différence significative $(P<0,05)$. Les fromages $R E$ et $N R$ contenaient les plus grandes quantités d'acides libres. Cela est vraisemblablement dû à la présence d'enzymes lipolytiques (lipase, esterase, etc.) dans les préparations commerciales de coagulants fongiques (Sternberg, 1976).

\subsection{Qualités organoleptiques des fromages}

Pour tous les fromages, le score final variait entre 95,1 et 98,7 points pour une durée d'affinage de 30 à 60 jours. Ce dernier progressait de façon régulière et les fromages atteignaient leurs meilleures caractéristiques 45 jours après leur fabrication (tab. 6). Quelques différences constatées au $20^{\mathrm{e}}$ jour de l'affinage, en ce qui concerne la saveur et l'arôme des fromages, disparaissaient 10 jours plus tard.

Tous les fromages étaient acceptables du $30^{\mathrm{e}}$ au $60^{\mathrm{e}}$ jours. Ils possédaient un goût agréable, piquant et une pâte molle, onctueuse 


\section{TABLEAU 6 - TABLE 6}

Evolution des qualités organoleptiques des fromages fabriqués à l'aide de présure animale (PR), de Noury Rennet (NR), de Rennilase (RE) et de Suparen (SU)

Evolution of the organoleptic characteristics of cheeses produced using calf rennet (PR), Noury Rennet (NR), Rennilase (RE) and Suparen (SU) as milk coagulants

\begin{tabular}{|c|c|c|c|c|c|}
\hline & \multirow{2}{*}{ Fromage } & \multicolumn{4}{|c|}{ Durée de l'affinage (jours) } \\
\hline & & 20 & 30 & 45 & 60 \\
\hline Saveur et arôme & $\begin{array}{l}\text { PR } \\
\text { NR } \\
\text { RE } \\
\text { SU }\end{array}$ & $\begin{array}{l}42,8 \mathrm{a} \\
42,3 \mathrm{ab} \\
41,9 \mathrm{~b} \\
41,9 \mathrm{~b}\end{array}$ & $\begin{array}{l}43,9 \\
43,7 \\
43,2 \\
43,2\end{array}$ & $\begin{array}{l}44,8 \\
44,7 \\
44,4 \\
44,3\end{array}$ & $\begin{array}{l}43,0 \\
43,2 \\
43,3 \\
42,8\end{array}$ \\
\hline Texture et fermeté de la pâte & $\begin{array}{l}\text { PR } \\
\text { NR } \\
\text { RE } \\
\text { SU }\end{array}$ & $\begin{array}{l}33,3 \\
33,5 \\
33,1 \\
33,6\end{array}$ & $\begin{array}{l}33,8 \\
33,8 \\
33,7 \\
33,8\end{array}$ & $\begin{array}{l}34,3 \\
34,5 \\
34,5 \\
34,6\end{array}$ & $\begin{array}{l}33,5 \\
33,4 \\
33,7 \\
33,5\end{array}$ \\
\hline Couleur et apparence générale & $\begin{array}{l}\text { PR } \\
\text { NR } \\
\text { RE } \\
\text { SU }\end{array}$ & $\begin{array}{l}13,8 \\
13,8 \\
13,8 \\
13,8\end{array}$ & $\begin{array}{l}14,2 \\
14,4 \\
14,4 \\
14,5\end{array}$ & $\begin{array}{l}14,6 \\
14,6 \\
14,5 \\
14,7\end{array}$ & $\begin{array}{l}14,2 \\
14,0 \\
13,8 \\
14,0\end{array}$ \\
\hline Sel & $\begin{array}{l}\text { PR } \\
\text { NR } \\
\text { RE } \\
\text { SU }\end{array}$ & $\begin{array}{l}4,9 \mathrm{a} \\
4,9 \mathrm{a} \\
4,9 \mathrm{a} \\
4,8 \mathrm{~b}\end{array}$ & $\begin{array}{l}4,9 \mathrm{a} \\
4,8 \mathrm{~b} \\
4,9 \mathrm{a} \\
4,8 \mathrm{~b}\end{array}$ & $\begin{array}{l}4,9 a \\
4,9 a \\
4,9 a \\
4,8 b\end{array}$ & $\begin{array}{l}4,9 a \\
4,8 b \\
4,9 a \\
4,8 b\end{array}$ \\
\hline
\end{tabular}

$\mathrm{a}, \mathrm{b}$ : les valeurs d'une même colonne, portant une lettre différente, diffèrent significativement au seuil de $\mathrm{P}=0,05$. 
et ils étaient couverts uniformément d'une couche abondante de Penicillium. Les fromages NR et SU étaient parfois plus doux que les témoins, mais les différences atteignaient à peine la plus petite différence significative au seuil 0,05 . Aucun défaut n'a été décelé.

\section{CONCLUSIONS}

La composition des fromages fabriqués à l'aide des protéases microbiennes différait de celle du fromage témoin. Leur $\mathrm{pH}$ était un peu plus bas, les produits de la protéolyse plus abondants. Le fait que, malgré les différences de la vitesse et du type de protéolyse et la nature différente des produits de dégradation, l'évolution du $\mathrm{pH}$ des fromages avait lieu de la même manière, confirme que la flore superficielle était le principal facteur de modification du $\mathrm{pH}$, par la consommation de l'acide lactique et la formation d'ammoniac et d'autres substances alcalines.

L'action de la présure et des coagulants fongiques, limitée sur les caséines et les peptides à haut poids moléculaire, ne pouvait pas influencer fortement les qualités organoleptiques des fromages qui atteignaient leur maturité après une période de temps identique.

La fabrication des fromages expérimentaux ne posait pas de problèmes et les rendements fromagers étaient semblables. Cela, ajouté à l'absence complète de défauts organoleptiques du produit final, permet de conclure que les extraits coagulants microbiens Noury Rennet, Rennilase et Suparen possèdent une bonne aptitude à la fabrication de fromage de chèvre à croûte fleurie.

\section{Bibliographie}

AlaIs (C.) et NovaK (G.) (1968). - Etude d'un enzyme coagulant microbien dérivé de Endothia parasitica. Le Lait, 48, 393.

Alais (C.) et Lagrange (A.) (1972). - Etude biochimique d'une protéase coagulante produite par Mucor miehei. I. Activité coagulante et protéolytique. Le Lait, 52, 407.

Assenat (L.) (1967), - Contribution à l'étude d'une méthode d'identification des laits et fromages au moyen de l'électrophorèse sur gel de polyacrylamide. Le Lait, 47, 393.

Dismazeaud (M. J.) et Juge (M.) (1976). - Caractérisation de l'activité protéolytique et fractionnement des dipeptidases et des aminopeptidases de Streptococcus thermophilus. Le Lait, 56, 241.

ExTERKATE (F. A.) (1975), - An introductory study of the proteolytic system of Streptococcus cremoris strain HP. Neth. Milk Dairy J., 29, 303.

FÉD. Int. LaIt. - Normes Internationales, 4: 1958 et 17 A : 1972.

FÉd. INT. LAIT. (1983). - Survey on the production and utilization of goat's milk. Doc. 158. 
Green (M. L.), StackPoole (A.) (1975). - The preparation and assessment of a suitable Mucor pusillus Lindt proteinase - swine pepsine mixture for Cheddar cheesemaking. J. Dairy Res., 42, 297.

Gripon (J. C.), Desmazeaud (M. J.), Le Bars (D.) and Bergère (J. L.) (1977). Role of proteolytic enzymes of Streptococcus lactis, Penicillium roqueforti, and Penicillium caseicolum during cheese ripening. J. Dairy Sci., 60, 1532.

IYer (M.), Richardson (T.), AMUdson (C. H.), and BoudREAU (A.) (1967). - Improved technique for analysis of free fatty acids in butteroil and Provolone cheese. J. Dairy Sci., 50, 285.

KosikowsKy (F.V.) (1951). - The liberation of free amino acids in raw and pasteurized milk Cheddar cheese during ripening. J. Dairy Sci., 34, 228.

Maubois (J.L.) et Mocouot (G.) (1969). - Action protéolytique d'un enzyme coagulant comparée à celle de la présure. Le Lait, 49, 497.

Mrowetz (G.) (1979). - Kolorimetrische Bestimmung des Ammoniumgehaltes von Käsen. Milchwissenschaft, 34, 593.

Nelson (J. A.) and Trout (G. M.) (1965). - Judging Dairy Products. 4th edition revised. The Olsen Publ. Co., Milwaukee (Wisconsin, U.S.A.).

Nelson (J.H.) (1969). - Commercial scale cheesemaking trials with a milk clotting enzyme produced by Mucor pusillus Lindt. J. Dairy Sci., 52, 889.

NieUWENHOF (F.F.J.) and HuP (G.) (1971). - Gas chromatographic determination of free fatty acids in cheese. Neth. Milk Dairy J., 25, 175.

Noomen (A.) (1983). - The role of the surface flora in the softening of cheeses with a low initial pH. Neth. Milk Dairy J., 37, 229.

RAMET (J.P.) et Alais (C.) (1972). - Etude d'une protéase coagulante produite par Mucor miehei. II. Utilisation de la Rennilase dans la fabrication de fromages à pâtes molles. Le Lait, 52, 654.

Ramet (J. P.), Alais (C.) et Weber (F.) (1969). - Etude d'un enzyme coagulant microbien dérivé de Endothia parasitica. II. Fabrications expérimentales de fromages à pâte molle et à pâte cuite avec l'enzyme coagulant Pfizer. Le Lait, 49, 40.

Spackman (D. H.), Stein (W. H.) and Moore (S.) (1958). - Automatic recording apparatus for use in the chromatography of amino acids. Anal. Chem., $30,1190$.

Sternberg (M.) (1976). - Microbial rennets. Adv. Appl. Microb., 20, 135.

Thomas (T. D.) and Mills (O. E.) (1981). - Proteolytic enzymes of starter bacteria. Neth. Milk Dairy J., 35, 255.

VANDERPOORTEN (R.) and WeckX (M.) (1972). - Breakdown of casein by rennet and microbial milk-clotting enzymes. Neth Milk Dairy J., 26, 47. 